\title{
Digital control of delivery and release of oil products at fuel depots and gas stations
}

\author{
Ilya Rebrov ${ }^{1,}{ }^{*}$, Sergey Naumenko ${ }^{1}$, Alexandr Godnev ${ }^{2}$, and Evgeny Kossov ${ }^{1}$ \\ 'JSC "VNIIZhT" (Railway Research Institute), Moscow, 129626, Russia \\ ${ }^{2}$ Scientific and Technical Center for Information and Measurement Technology ("STC IMT" LLC), \\ Korolev, 141070, Russia
}

\begin{abstract}
The paper shows the topicality of the transition from the metering system for oil products at fuel depots and gas stations, organized with the help of automated measuring instruments, to the use of automated information and measurement systems for oil products metering (IMS OP) of a new generation. The structure diagram of the IMS OP is presented, the implementation of which allows solving the problem of formation of the normalized value of the unbalance between the actual results of measuring the mass of OP in the technological chain "Supplier - Distribution system Consumer" and the current document flow. The basic algorithm of IMS OP operation is presented, as well as the results of its approbation as a part of IMS OP at the fuel depot of the Dno station of the October Railway.
\end{abstract}

\section{Introduction}

Everyone knows from school the problem of pouring water into the pool from the supply pipe and draining it through the discharge pipes. Obviously, the volume of water in the pool should be the difference between the volume of water supplied into the pool and the volume of water withdrawn from the pool. A similar problem is being solved at the fuel depots of JSC "Russian Railways", where the inventory commission carries out measurements of the residual quantities of oil products (OP) in the tanks that are filled and drained within a month.

The complexity of performing measurements is that it is necessary to determine not the volume, as in the school problem, but the mass of OP, which requires knowledge of the value of the density of $\mathrm{OP}-\rho$, which in turn depends on its constantly changing temperature, i.e.

$$
M=\rho(t) \times V,
$$

where: $M \quad-\quad$ mass of oil product, $\mathrm{kg}$;

$\rho(t) \quad-$ density of oil product depending on its temperature, $\mathrm{kg} / \mathrm{m}^{3}$;

$V \quad-\quad$ volume of oil product, $\mathrm{m}^{3}$,

and also have accurate calibration characteristics of tanks.

Taking into account the difficulty of the problem being solved, GOST R8.595-2004 allows the value of the mass of OP measured during inventory to deviate from the

${ }^{*}$ Corresponding author: Rebrov.Ilya@vniizht.ru 
corresponding value specified in accounting documents by not more than $0.5 \%$ [1]. Thus, the shortage of OP in the amount of less than $0.5 \%$ detected during the measurement can be attributed by the inventory commission to the standardized error of measuring instruments. A shortage exceeding $0.5 \%$ must be recovered either from the financially responsible person or be attributed to the losses of the fuel depot. Here it should be noted that GOST [1] also allows the measured value of the mass of OP to differ from the mass specified in waybill both when it is delivered to the fuel depot and when it is released from the fuel depot.

All these assumptions in the chain: shipment of OP from the Supplier, acceptance at the fuel depot, storage, release, and inventory do not allow predicting unambiguous reduction of the commodity balance of OP. For example, the supplier (Oil refinery) shipped to the customer (Roszheldorsnab - branch of JSC "RZD", then the distribution system) 1,000 t of OP according to the results of measurements of the weighing unit, the relative error of which is $\pm 0.4 \%$. Let us assume that the absolute error of measurements was 2.0 tons, i.e. $0.2 \%$. Also assume that the customer released to the consumer (diesel locomotives of the Directorate of traction, track machines belonging to the Directorate of infrastructure, third-party consumers ...) $900 \mathrm{t}$ of OP with "zero error". Then, in the customer's accounting documents, the value of the residual $M=1000-900=100$ tons, and the actual mass of OP in the tank will be 998 - $900=98$ tons, i.e. in relation to accounting documents, there is an unbalance of 2 tons. When inventorying, the commission has the right to assign $\pm 0.5 \%$, i.e. 0.5 tons from 100 tons, to the standardized value of the error of measuring instruments for the tank, and the redemption of a shortage of $1.5 \mathrm{t}$ must be presented to the financially responsible person or attributed to the losses of JSC "RZD". The aim at switching to automated metering systems of OP [2], involving the equipping of fuel depots with more accurate automated measuring instruments (MI), does not lead to the exclusion of commodity unbalance of OP, but only increases the accuracy of metering and minimizes losses associated with possible unauthorized withdrawal of OP.

To reduce the commodity balance of OP at fuel depots, it is necessary to use specialized information and measurement systems for oil products metering (IMS OP), which are based on the adaptive algorithm for monitoring and metering of OP [3].

\section{Materials and methods}

In order to ensure that the unbalance is always within the specified normalized limits, it is necessary to distribute the unknown mass of the supplier's OPs $M_{0}$ in proportion to each small dose of the OP released to the consumer [4-6].

To fulfill this condition, the result of the measurement of the OP mass made by supplier $M_{0}$ and recorded in the waybill and the accounting data of the document flow of the Distribution System is taken as the actual value, which in this particular task is equated to the true value. The volume of the tank which is occupied by the mass of OP $M_{0}$ is divided into $\mathrm{n}$ "reference" volumes of $\Delta V_{p}$ (quanta), each of which is assigned the conditional value of the mass of OP

$$
m_{p}=M_{0} / n
$$

When one quanta $\Delta V_{p}$ with the conditional mass $m_{i p}$ is removed from the tank, a comparison is made with the result of measurements of this conditional mass by the metering unit $m_{\text {iun. }}$

In the case of inequality of the measurement results $m_{i p} \neq m_{i u n}$, the following results of measurements of the released quanta by the metering unit $m_{(i+1) u n}$ are corrected by some value $K_{i c}$ standardized in certain limits

$$
m_{(i+1) p}=m_{(i+1) u n}\left(1+K_{i c}\right)
$$

where 


$$
K_{i c}=\left|\frac{m_{i p}-m_{\text {iun }}}{m_{\text {iun }}}\right| \leq K_{\text {lim }},
$$

$K_{i}$ lim - limiting coefficient of correction of the measurement result of the metering unit.

Since GOST R.595-2009 regulates the limiting relative error of the OP supplier $\delta M_{0}= \pm$ $0,5 \%$, and the limiting relative error of the metering unit, as a rule, is within $\delta m_{\text {iun }} \leq \pm 0,15 \div$ $0,25 \%$, then the correction limit of the result measurements of the metering unit in the waybill when the mass of OP is released to the consumer, as well as by the mass to be written off from the IMS database, will be determined by the formula (5)

$$
K_{\lim }(\%) \approx \pm \sqrt{\delta \mathrm{M}_{\mathrm{o}}^{2}-\delta m_{\text {iun }}^{2}},
$$

assuming that $\delta \mathrm{M}_{0}$ and $\delta \mathrm{m}_{\mathrm{iy}}$ are independent values.

For example, for $\delta m_{\text {iun }} \approx \pm(0,15 \div 0,25 \%)$, we have

$$
K_{\text {lim }} \approx \pm \sqrt{0,5^{2}-\left(0,15^{2} \div 0,25^{2}\right)}= \pm(0,476 \div 0,443 \%)
$$

In this case, the relative error in the release of OP by mass to the consumer will always be in the normalized range of $\pm 0.5 \%$.

The correction coefficient of the first quanta $K_{\text {Icor }}$ is calculated by the formula (6)

$$
K_{l c}=1+\frac{\mathrm{M}_{0}-\mathrm{M}_{a p}}{\mathrm{M}_{a p}} \leq 1+\left(0 \pm \mathrm{K}_{\text {lim }}\right)
$$

where $M_{a p}$ - the result of measurements of the actual mass of OP along the tank before starting its emptying.

\section{Results}

Fig. 1 shows a structure diagram of the IMS OP. The principle of operation of the IMS OP system is as follows.

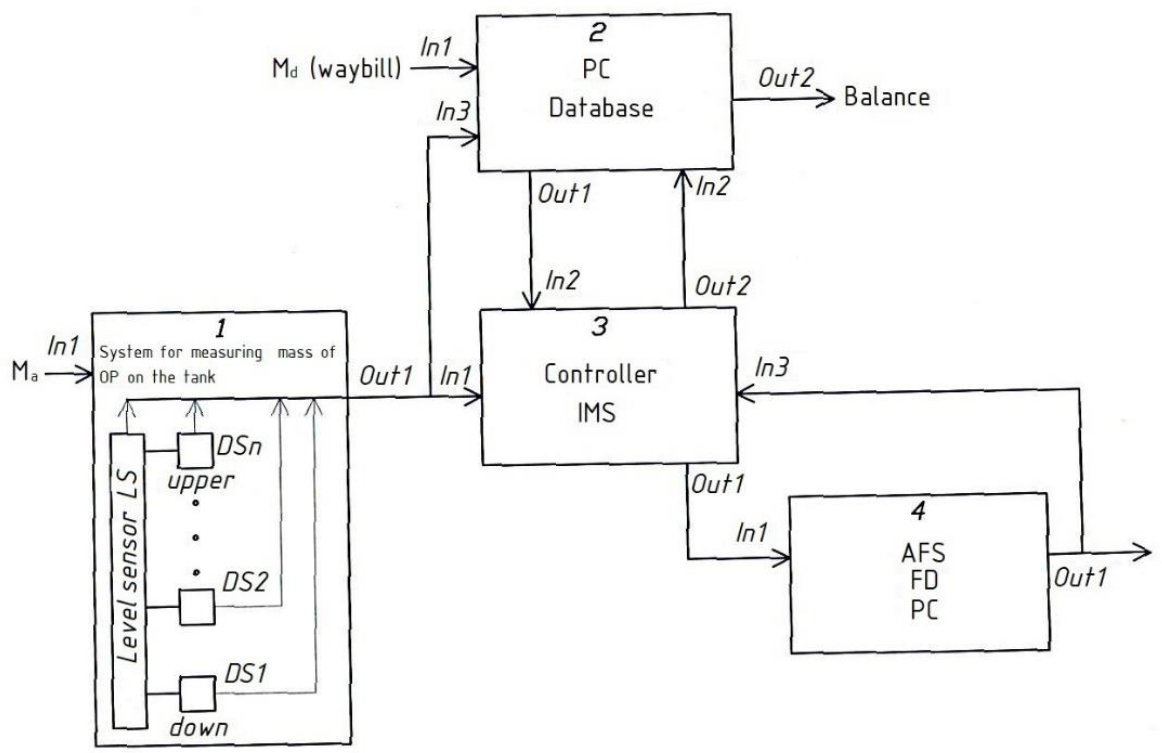

Fig. 1. Structural diagram of information and measurement system of oil products metering.

When the OP is received, the actual mass of oil products $M_{a r}$ is received at input 1 of system 1 . At the output of system 1 , the result of measurements $M_{a p}$ is generated, which is 
fed to the input 1 of the computer 3 . The input 3 of the database 2 receives the information of the supplier's result of measurements of the mass of OP delivered to the customer. The information from the output 1 of the database 2 goes to the input 2 of the computer 3 . The command for the release of OP comes from the output 1 of the computer 3 to the input of the metering unit 4 (automatic filling stand or fuel dispenser). The result of the measurement of the released mass of OP comes to the input 3 of the computer 3 . The computer 3 compares the two masses of OP: left from the tank of the system 1 and released by the metering unit 4 . At the output 2 of the computer 3, a corrected measurement result is produced, which is released by the metering unit 4 of the OP mass. The program of the computer 3 recorded the level passing through one end gauge, then, as soon as OP is released, through the second, etc., and compares it with the results (volume, density, mass) obtained from the measuring unit 4 . The computer 3 calculates the correction coefficient for the measurement result of the mass of the quanta $m_{i q}$ by the metering unit 4 and corrects it. The corrected result from the output 2 of the computer 3 goes to the input 2 of the database 2 . The input 3 of the database 2 receives the measurement result from the output 1 of the system 1 , which shows the actual value of the residual mass of OP in the tank $M_{p \text {.res }}$ after the release.

At the output 2 of the database 2 , the current result of the unbalance is formed, which is equal to the difference between the actual results of measurements of the mass of the OP in the tank $M_{\text {a.res }}$ and the residual mass in the database $M_{\text {d.res }}$.

In general, the above algorithm for synchronizing the results of measurements at the tank and metering unit allows minimizing the amount of unbalance within $\pm 0.1 \%$, provided that the initial mass of OP $M_{a p}$ in the tank does not differ from the measurement result of the supplier by more than $\pm 0.4 \%$, and the metering unit does not exceed the limits of the relative error $\pm 0.25 \%$.

\section{Discussion}

Fig. 2 presents a graph of approbation of the above unbalance control algorithm for the release of diesel fuel, which was implemented in 2011 at the fuel depot of the Dno station of the October Railway.

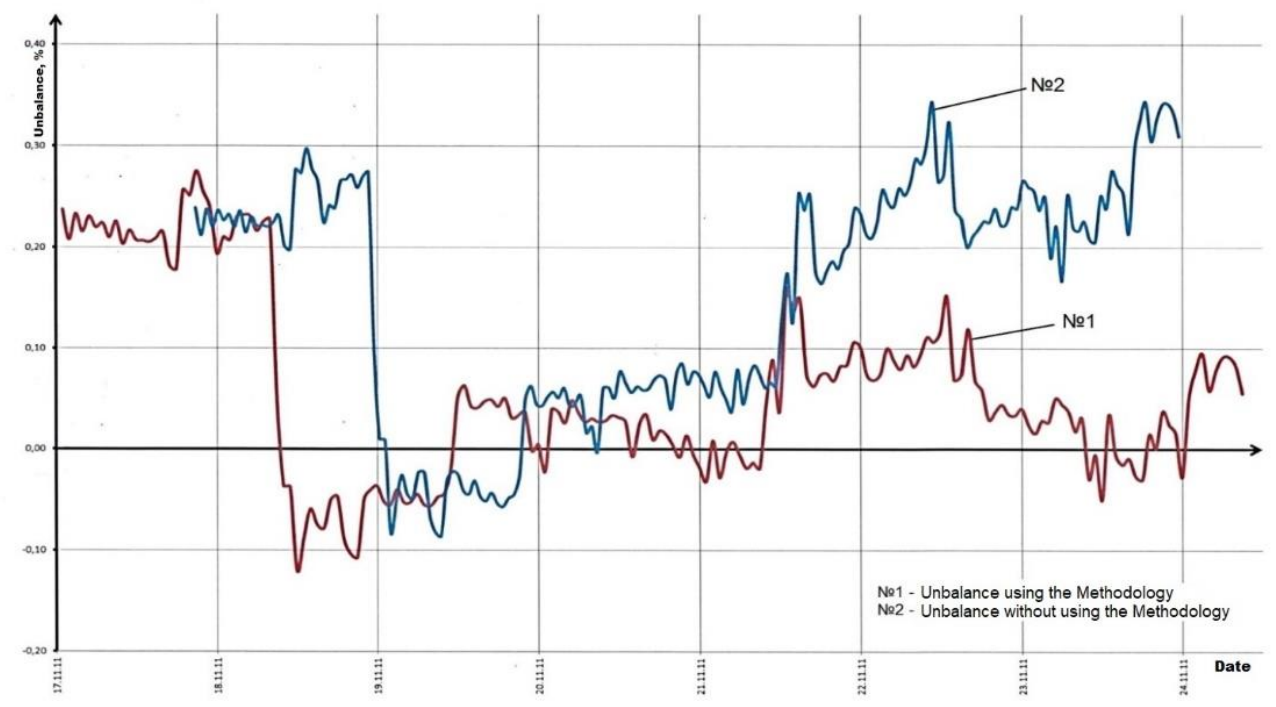

Fig. 2. Approbation of the adaptive algorithm of control of unbalance at the fuel depot of the Dno station of the October Railway. 
Graph № 1 shows that the control loop of the IMS OP tends to compensate for the possible shortage of diesel fuel to the tank within $\pm 0.1 \%$ in relation to the database residuals. At the same time, the graph № 2 shows a confident positive trend of the unbalance, which characterizes the accumulation of surplus diesel fuel in the tank in relation to the document flow. Thus, initially, as a part of the approbation, the fuel dispenser was set to constantly underfill to the consumer within $\approx-0.3 \div 0.4 \%$. Thus, the introduction of IMS ensures the optimal transfer of the volume of OP to the consumer within the uncertainty of measurement of the mass of OP $\pm 0.5 \%$, existing in accordance with GOST R 8.595-2004, when receiving, storing and releasing. This eliminates the principal possibility of adjusting the metering unit or fuel dispenser to a permanent underfill (introducing a negative systematic error) not only over $-0.5 \%$ but within the allowable error range of $\pm 0.5 \%$. It should also be noted that if there is a surplus of OP in the tank within $+0.5 \%$, they will be transferred to the Consumer by the IMS OP system $[7,8]$.

\section{Conclusions}

1. An effective way to maintain the normalized value of the unbalance between the results of oil product mass measurements by the information and measurement system and the corresponding document flow while it is moving in the technological chain Supplier Distribution system - Consumer is developed.

2. A structural diagram and corresponding software have been developed that implement the algorithm for the adaptive functioning of the IMS OP and reducing the commodity balance when conducting accounting operations within the uncertainty of the results of measuring the mass of oil products by the Distribution system when they are received, stored and released. 3. The quantitative agreement of the developed algorithm for the operation of the IMS OP with experimental data is obtained.

4. Implementation of the IMS OP allows solving a large inter-industry problem of formation of the normalized value of the unbalance between the actual results of measurements of the oil product's mass in the technological chain "Supplier-Distribution system-Consumer" and the current document flow.

\section{References}

1. Russian Federation Standard GOST R8.595-2004

2. L. Zolotareva, Point of support 191, 3 - 5 (2014)

3. A.G. Godnev, S.N. Naumenko, Railway Research Institute Bulletin 5, 44-47 (2010)

4. S.N. Naumenko, A.G. Godnev, Railway transport 2, 49-50 (2011)

5. A.G. Godnev, N.V. Davydov, S.N. Naumenko, Patent 2495818, Method of reducing the commodity balance at oil depots and gas stations during the reception, storage and release of oil products in oil product supply systems and the system for its implementation (2013)

6. A.G. Godnev, E.P. Loktev, S.N. Naumenko, Railway Research Institute Bulletin 5 (2016)

7. S.N. Naumenko, A.G. Godnev, Railway transport at the present stage, collection of works of scientists to the 70th anniversary of postgraduate study (VMG-Print, Moscow, 2014)

8. Methodology for automated reduction of commodity balance of the oil product at the fuel depot of JSC “Russian Railways” № 1537 r. (2012) 